Research Paper

\title{
'H NMR Based Serum Metabolic Profiles Associated with Pathological Progression of Pancreatic Islet $\beta$ Cell Tumor in Rip1-Tag2 Mice
}

\author{
Yongxia Yang 1,2, Ying Liu1,2, Lingyun Zheng1, Qianqian Zhang², Quliang Gu1 ${ }^{1}$, Linlin Wang1, Lijing \\ Wang ${ }^{\circledR}$ \\ 1. School of Basic Course, Guangdong Pharmaceutical University, Guangzhou, 510006, PR China \\ 2. Vascular Biology Research Institute, Guangdong Pharmaceutical University, Guangzhou, 510006, PR China \\ $\triangle$ Corresponding author: Prof. Lijing Wang: Vascular Biology Research Institute, Guangdong Pharmaceutical University, Guangzhou, \\ 510006, P. R. China; Tel: 86-(0)20-3935-2126, Fax: 86-(0)20-3935-2126, Email: sheepling-2004@163.com.
}

(c) 2015 Ivyspring International Publisher. Reproduction is permitted for personal, noncommercial use, provided that the article is in whole, unmodified, and properly cited. See http://ivyspring.com/terms for terms and conditions.

Received: 2014.11.14; Accepted: 2015.02.28; Published: 2015.04.05

\begin{abstract}
Pancreatic islet $\beta$ cell tumor is the most common islet cell tumor. A well-characterized tumor progression in Ripl-Tag2 mice undergoes five stages, involving normal, hyperplasia, angiogenic islets, tumorigenesis and invasive carcinoma. 'H NMR based metabonomics was applied to identify potential biomarkers for monitoring pancreatic islet $\beta$ cell tumor progression in Ripl-Tag2 mice. Multivariate analysis results showed the serum metabonome at hyperplasia stage shared the similar characteristics with the ones at normal stage as a result of slight proliferation of pancreatic islet $\beta$ cells. At angiogenic islets stage, the up-regulated glycolysis, disturbed choline and phospholipid metabolism composed the metabolic signature. In addition to the changes mentioned above, several metabolites were identified as early biomarkers for tumorigenesis, including increased methionine, citrate and choline, and reduced acetate, taurine and glucose, which suggested the activated energy and amino acid metabolism. All the changes were aggravated at invasive carcinoma stage, coupled with notable changes in alanine, glutamate and glycine. Moreover, the distinct metabolic phenotype was found associated with the implanting of SV40 large T antigen in Rip1-Tag2 mice. The combined metabolic and multivariate statistics approach provides a robust method for screening the biomarkers of disease progression and examining the association between gene and metabolism.
\end{abstract}

Key words: ${ }^{1} \mathrm{H}$ NMR; Metabonomics; Pancreatic islet $\beta$ cell carcinoma; Rip1-Tag2; Serum; Multivariate analysis

\section{Introduction}

Pancreatic islet $\beta$ cell tumor, namely insulinoma in clinical, occupies a proportion of $70-80 \%$ of pancreatic tumor [1,2]. Clinical incidence of insulinoma is about 3-4 per million in the world, presenting a female preponderance [3]. Insulinoma can occur in large scope of ages ranging from 8 to 82 years old with a median age of 47 years [3]. Commonly, insulinomas locates in intra pancreatic with $10 \%$ of malignancy [3]. Although the 10 years' survival rate of insulinomas is about 88 percent after excision, the lesions less than 2 $\mathrm{cm}$ account for $90 \%$ and the tumor diameter in $50 \%$ of patients is less than $1.3 \mathrm{~cm}$ [4]. So the early diagnosis for insulinoma is still a challenging work for clinical practice. Hypoglycaemia is the secondary common symptom in addition to excessive insulin secretion [4]. Chronic hypoglycaemia can definitely result in death. The delayed or mistaken diagnosis of hypoglycaemia and other common symptoms always increase the severity and mortality of insulinomas. Therefore, it is important to early diagnose to reduce the severity and mortality rate.

There are many diagnosis techniques for insu- 
linomas, such as transabdominal ultrasonography, EUS, MRI and CT, etc. Arteriography is rarely used in clinical diagnosis due to its low sensitivity and invasion $[5,6]$. The high sensitivity ranging from 57 to 94 percent has enabled the EUS popular in recent years $[7,8]$. But it has many limitations in evaluation of malignancy and poor differentiation of adjacent lesions [9]. The diagnostic sensitivity of CT is also increased as the advent of new advanced technology, and the sensitivity of dual-phase multidetector CT is up to 94 percent though false-negative results are often induced [8]. MRI is an accurate tool for detecting the small pancreatic insulinomas and outside metastasis with high sensitivity, but the fee is high and more time is required. For the techniques mentioned above, the early diagnosis, when the tumor nodules are in size less than $1 \mathrm{~mm}$, is often difficult. Moreover, the biochemical information at molecular level, which can distinguish the pathological tissues from the adjacent tissues, rarely can be provided from the aforementioned techniques.

Metabonomics, as a part of systems biology, has been used to determine the biochemical changes caused by intrinsic and external factors [10,11]. In recent years, metabonomics has been widely used for screening of early metabolic biomarkers in numerous diseases, especially in tumors, such as liver [12], breast [13], prostate [14], pancreas [15], kidney [16], colorectal [17] cancers. Magnetic resonance spectroscopy (MRS) as an important analytical technique has been widely used in metabonomics researches [18].

Rip1-Tag2 transgenic mouse is one of animal models of islet $\beta$ cell tumor, which has been frequently used in studying tumor progression [19], inflammation [20], regulation of signal pathway [21,22] and therapeutic evaluation [23]. SV40 large T antigen oncogenes are transplanted to islet cells through an insulin gene promoter, and so some tumor suppressor factors were devitalized and oncogenesis was induced in islet cells [20]. A stable and reproducible tumor development in Rip1-Tag2 mice undergoes normal (about 1-3 weeks), hyperplasia (about 4-5 weeks), angiogenic islets (about 6-8 weeks), tumor (about 9-10 weeks) and invasive carcinoma (about 11-14weeks) [22,24,25]. The disease progression in Rip1-Tag2 mice is similar with the development of insulinoma in human, and thus this model can be used in detecting dynamic progression and screening out the early diagnostic biomarkers for insulinomas.

In this report, we presented the serum metabolic characteristics of Rip1-Tag2 transgenic mice at five stages to screen out the biomarkers for pathological progression in pancreatic islet $\beta$ cell cancer, with a view to provide the early diagnostic biomarkers for insulinoma. Pattern recognition analysis was also ap- plied to draw the metabolite characteristics that were specifically associated with implanting of SV40 large $\mathrm{T}$ antigen.

\section{Materials and methods}

\section{Rip1-Tag2 mice identification and sample col- lection}

The Rip1-Tag2 mice [B6.D2-Tg(RIP1-Tag2)2Dh] were obtained from Institute of Biochemistry and Cell Biology, SIBS, CAS and were crossed with C57 mice (C57/BL6, Rip1-Tag2 background mice), purchased from Animal Center of Guangdong province (Guangzhou, China). To obtain the targeted Rip1-Tag2 transgenic mice, we identified the gene type with PCR technology. The targeted Rip1-Tag2 product size was about 500bp, shown in lane 1-6 and 10 (Supplementary Material: Fig. S1). Correspondingly, the 7-9 lanes without 500bp mark represented the background mice $\mathrm{C} 57$, as negative control in this study.

Mice were housed in an animal house with controlled temperature $\left(23-24{ }^{\circ} \mathrm{C}\right)$ and humidity $(50 \pm 10 \%)$, on an average light/dark cycle. Rip1-Tag2 mice and C57 mice used in this study were all littermates with no difference between male and female. This research was approved by the Committees for Ethical Review of Research involving Animal Subjects at Guangdong Pharmaceutical University, China. The Rip1-Tag2 mice at 3, 5, 8, 10 and 14 weeks were sacrificed at the indicated time. Blood (approximately 1.5 $\mathrm{ml}$ ) were collected for serum samples and pancreases and tumor tissues were dissected for histopathological examination.

\section{HE staining}

The fresh tissues were separated and fixed in $4 \%$ formalin overnight. After dehydration for about 9 hours, the tissues were embedded in paraffin immediately. Then $4 \mu \mathrm{m}$ sections were cut and rehydrated after drying in $65^{\circ} \mathrm{C}$ dryer for 2 hours. Hematoxylin and eosin were stained after gradient dewaxing.

\section{Sample preparation}

After the serum samples dissolving, $300 \mu \mathrm{L}$ of each sample was diluted by $150 \mu \mathrm{L}$ PBS $(0.2 \mathrm{M}$, $\mathrm{pH}=7.4)$. The supernatants were transferred into NMR analysis tubes after centrifuging (5000 rpm, 10 $\left.\min , 4^{\circ} \mathrm{C}\right) .80 \mu \mathrm{L}_{2} \mathrm{O}$ was also added to each tube for the deuterium lock.

\section{NMR experiment}

Sera ${ }^{1} \mathrm{H}$ NMR spectrum was collected on Bruker spectrometer (Avance III $500 \mathrm{MHz}$ ). The experimental temperature is $298 \mathrm{~K}$. The pulse sequence is CPMG (relaxation delay-90-( $(-180-\tau)_{n}$-acquisition), which was 
applied to acquire the signals with low molecular weight selectively, with $2 \mathrm{nt}$ of $100 \mathrm{~ms}$ and relaxation delay of $2.5 \mathrm{~s} .128$ scans were collected, data points is $32 \mathrm{k}$ and spectral width is $10 \mathrm{kHz}$.

For signal assignments, 2D spectra of COSY, TOCSY and HSQC were acquired. In COSY and TOCSY experiments, 80 scans with 2048 data points were collected. A MLEV-17 programme was involved in TOCSY experiment with $90 \mathrm{~ms}$ mixing time. The spectral width for two dimensions is $10.0 \mathrm{ppm}$. In HSQC spectra, 120 increments were acquired into 2048 data points, the spectral width for ${ }^{1} \mathrm{H}$ and ${ }^{13} \mathrm{C}$ is $10.0 \mathrm{ppm}$ and $150.0 \mathrm{ppm}$, respectively.

\section{Date processing and multivariate analysis}

All FID data were Fourier transformed using Bruker Topspin 2.1 software (Bruker, Inc.). ${ }^{1} \mathrm{H}$ CPMG spectra at $\delta$ 0.50-9.50 were bucketed and automatically integrated using AMIX software (Bruker Biospin, Germany) with integration step of $0.005 \mathrm{ppm}$. The integral of residual water signal at $\delta$ 4.50-5.20 were set to zero. Then the integrals were normalized to the total integrals in one ${ }^{1} \mathrm{H}$ spectra.

Pattern recognition analysis was done using SIMICA-P+ (Umetric, Umea, Sweden). Intrinsic clusters according to the NMR spectra data was observed by PCA. Sequently, OPLS-DA was performed to establish the group separation for biomarker screening. The scale pattern is unit variance. The results of pattern recognition analysis were visualized by scores plot and loadings plot, where groupings pattern and metabolites differences were shown. The correlation coefficients from OPLS-DA were used to color the variables in loadings plots using a MATLAB programme. The significance of metabolites was further tested by a simple Student's test (SPSS 19.0).

\section{Results}

\section{Histopathological evaluation of pancreatic islet $\boldsymbol{\beta}$ cell tumor}

Fig. 1 showed a progressively histopathological assessment in Rip1-Tag2 mice. It was found that proliferation of pancreatic islet $\beta$ cells started at about 5 weeks with distinctly accumulated islet cells and enlarged volume compared to the morphological feature at 3 weeks. At about 8 weeks, angiogenic islets characterized by endothelial proliferation, vascular dilation, and microhemorrhagin was prevalent and then solid tumors emerged after soon. From the morphological feature at 10 weeks, we can find that the tumors were encapsulated and clear boundary from the surrounding tissue appeared, with a little tendency of microinvasion. Up to 14 weeks, invasive carcinoma commonly appeared in features of obvious atypia of islet structure and invasiveness with surrounding tissue. And both of tumor and invasive carcinoma were intensively vascularized by dilated blood vessels.

\section{Serum 'H CPMG Spectroscopy}

Five typical sera ${ }^{1} \mathrm{H}$ CPMG spectra from $\mathrm{C} 57$ (Fig. 2A) and Rip1-Tag2 mice at hyperplasia (Fig. 2B, 5 weeks), angiogenic islets (Fig. 2C, 8 weeks), tumor (Fig. 2D, 10 weeks) and invasive carcinoma (Fig. 2E, 14 weeks) stages were shown in Fig. 2, respectively. There are nearly no variability in metabolite composition and chemical shift perturbation among these spectra. The peaks were assigned on the basis of the published data [26-27] and determined by two dimension spectroscopy. The CPMG pulse sequence highlighted the smaller molecules through editing out those broad resonances. No obvious differences between the five groups were found visually due to the individual variability. Therefore, all the samples must be analyzed together by use of multivariate data analysis in order to get meaningful metabolites for different groups.
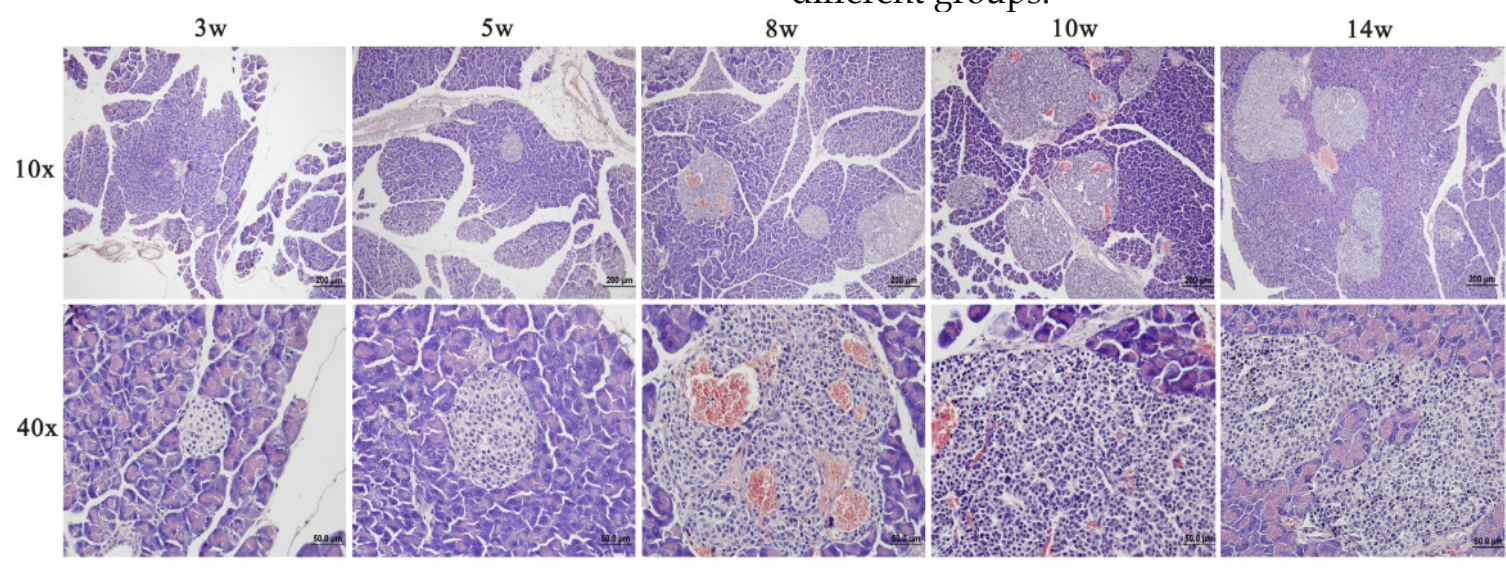

Fig. 1 Representative images of HE stainings of pancreatic and tumor tissues from Ripl-Tag2 mice at different stages (3, 5, 8, 10 and 14 weeks). 


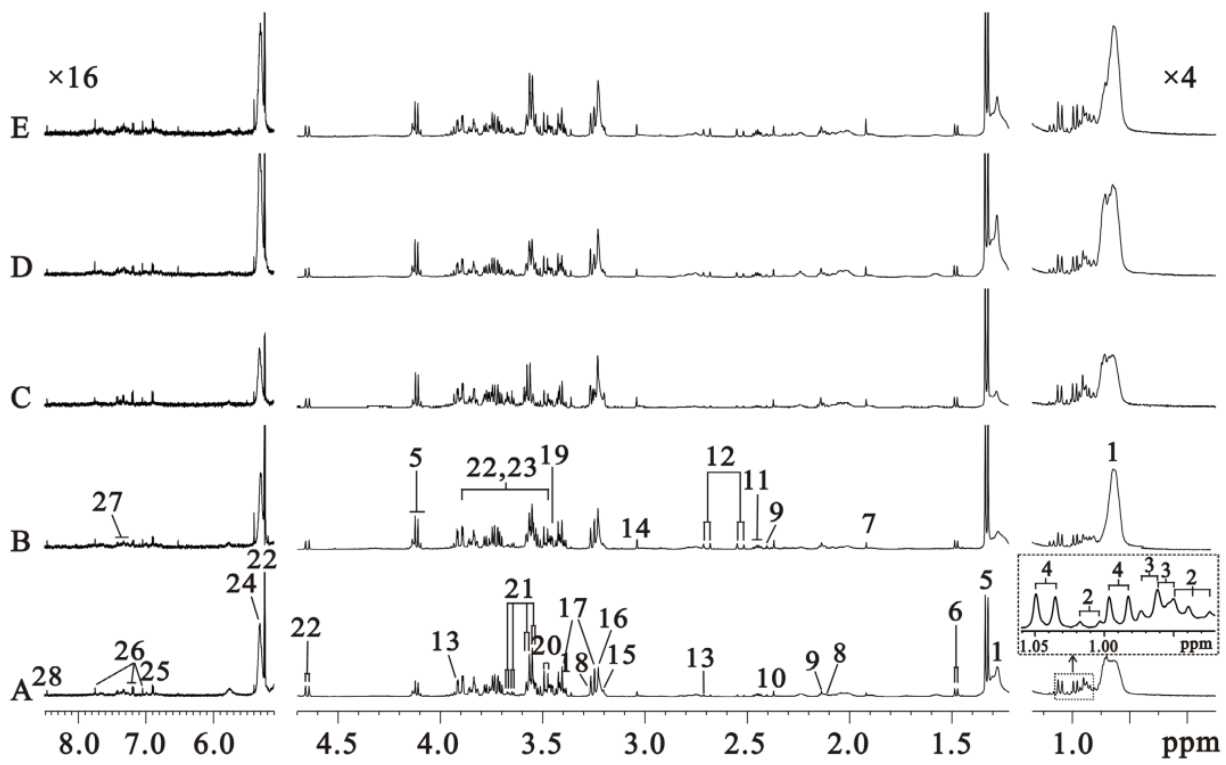

Fig. 2 500MHz sera 'H CPMG NMR spectra from C57 (A, 3 weeks) and Rip1-Tag2 mice (B, 5 weeks; C,8 weeks; D, 10 weeks; E, 14 weeks). Note: 1. lipid (mainly LDL and VLDL); 2. isoleucine (Ile); 3. leucine (Leu); 4. valine (Val); 5. lactate (Lac); 6. alanine (Ala); 7. acetate (Ace); 8. methionine (Met); 9.glutamate (Glu); 10. pyruvate (Pyr); 11.glutamine (Gln); 12.citrate (Cit); 13.dimethylamine (DMA); 14. creatine (Cr); 15.choline (Cho); 16. glycerophosphocholine + phosphocholine (GPC+PC); 17. taurine (Tau); 18. Trimethylamine-N-oxide (TMAO) and Betaine (Bet); 19. glycine (Gly); 20. threonine (Thr); 21. myo-inositol (MI); 22. a-glucose ( $\alpha$-Glc); 23. $\beta$-glucose ( $\beta$-Glc); 24. unsaturated lipid; 25. tyrosine (Tyr); 26. 1-methylhistidine; 27. phenylalanine; 28. formate.
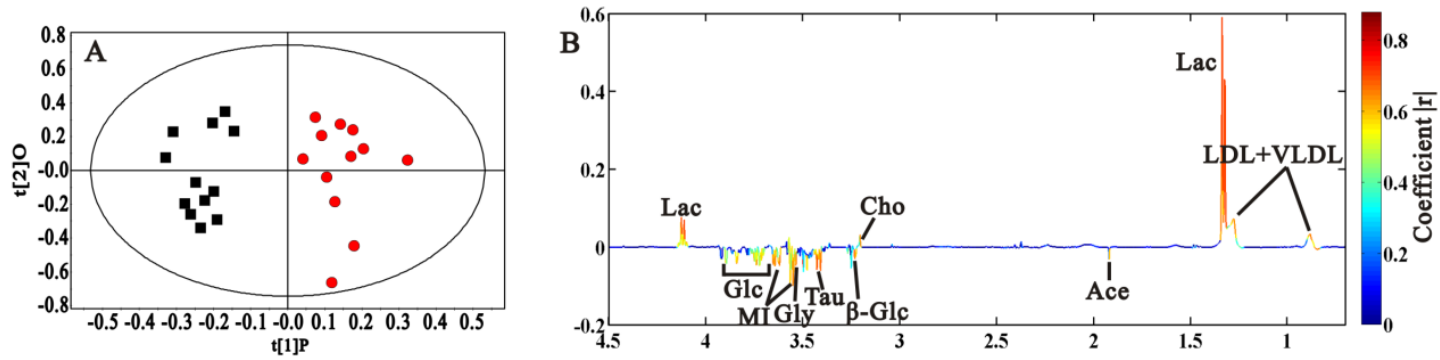

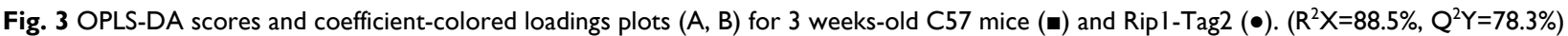

\section{Sera metabolic phenotype in Rip1-Tag2 mice}

We compared the sera metabolic profiles in morphologically normal Rip1-Tag2 mice (3 weeks old) and background mice of C57, in order to discover the metabolic difference induced by SV 40 gene transplantation. PCA was firstly done according to the ${ }^{1} \mathrm{H}$ NMR integral data at $\delta 0.5-4.5$ in order to highlight the contribution of metabolites with low molecular weight (in Supplementary Material: Fig. S2), in which obvious separation between these two types of mice was observed, demonstrating the distinct metabolic phenotype associated with SV 40 gene transplantation in Rip1-Tag2 mice. Clear grouping between Rip1-Tag2 and C57 through $t_{1}$ dimension was also found in OPLS-DA model (Fig. $3 \mathrm{~A}, \mathrm{R}^{2} \mathrm{X}=88.5 \%$, $\mathrm{Q}^{2} \mathrm{Y}=78.3 \%$ ). We can observe that lactate, LDL+VLDL and choline increased, and acetate, glucose, taurine, glycine and myo-inositol decreased in Rip1-Tag2 transgenic mice (Fig. 3B). All the selected metabolites showed statistical significance between Rip1-Tag2 and C57 mice, which may serve as biomarkers associated with SV 40 gene transplantation in Rip1-Tag2 mice (Table 1).

\section{Metabolic Variations Associated with Tumor Progression}

The PCA model was constructed to classify the Rip1-Tag2 sera samples at different stages. In Fig. 4, an obvious distribution trajectory was displayed with $\mathrm{R}^{2}$ of $82.8 \%$ and $\mathrm{Q}^{2}$ of $79.3 \%$ as the arrow showed. It was noted that there was serious overlapping between the samples at 3 weeks and 5 weeks. By looking at the ${ }^{1} \mathrm{H}$ spectra of these two groups, we speculated that this distribution pattern came from the similarity in ${ }^{1} \mathrm{H}$ spectra profiles. This original differentiation tendency demonstrated the dynamic metabolic variations correlated with the development of pancreatic islet $\beta$ cell carcinoma. Therefore, the serum metabolic characteristics reveal the metabonomic perturbations along with the pathological progress. 


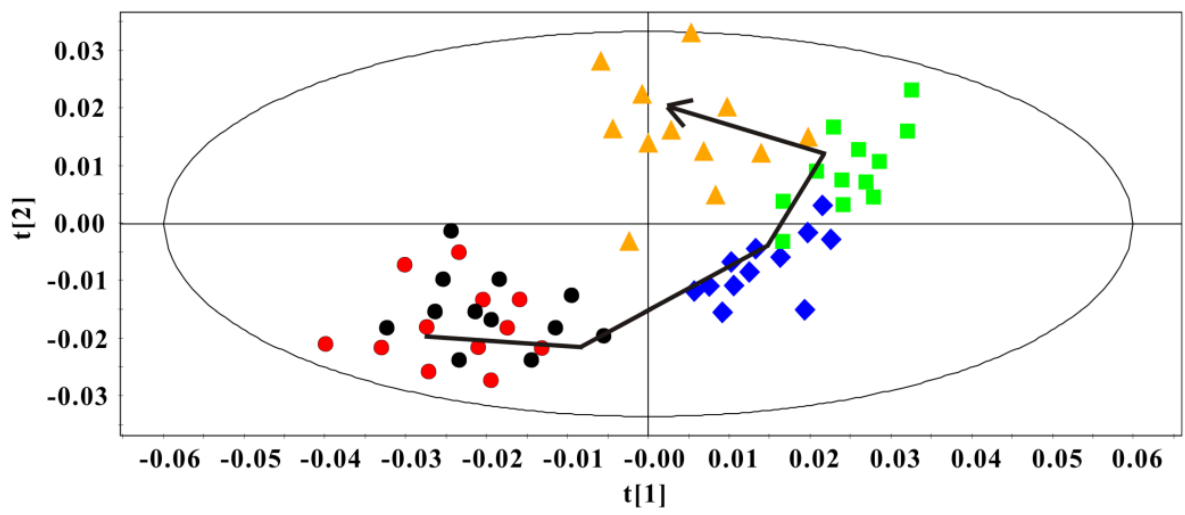

Fig. 4 PCA Scores plot of sera 'H NMR data in Ripl-Tag2 mice at different pathological stage $\left(R^{2}=82.8 \%, Q^{2}=79.3 \%\right)$. 3 weeks $(\bullet), 5$ weeks $(\bullet), 8$ weeks $(\diamond), 10$ weeks $(\boldsymbol{\square})$ and 14 weeks $(\boldsymbol{\Delta})$.

Table 1. Sera metabolites changes in Rip1-Tag2 mice at 3 weeks.

\begin{tabular}{lll}
\hline Metabolites & $\delta^{1} \mathrm{H}(\mathrm{ppm})$ & Rip1-Tag2 vs C57 \\
\hline LDL/VLDL & $0.89,1.27$ & $\uparrow^{*}$ \\
Ace & 1.92 & $\downarrow^{*}$ \\
Lac & $1.33,4.11$ & $\uparrow^{* *}$ \\
Cho & 3.20 & $\uparrow^{*}$ \\
Glc & $3.40-3.90$ & $\downarrow^{*}$ \\
Tau & 3.43 & $\downarrow^{* *}$ \\
Gly & 3.57 & $\downarrow^{*}$ \\
MI & $3.53,3.63$ & $\downarrow^{*}$ \\
\hline
\end{tabular}

$\uparrow$, denotes increased changes; $\downarrow$, denotes decreased changes; -, no statistical change " in comparison with C57." $p<0.05 ; "$ " $p<0.01$.

In this research, OPLS-DA models were built among the samples at different stages to find the potential biomarkers characterizing the histopathological progression. Scores and loadings line plots coded with coefficients were presented. The metabolites with significant contribution to the differentiation between two groups were hot colored. We didn't perform multivariate analysis on the spectra data at 5 weeks because of the metabolic similarity with the ones at 3 week. Fig. 5A1 showed the distinct grouping cluster between samples at 3 weeks and the ones at 8 weeks, which revealed the obvious sera metabolic changes in Rip1-Tag2 mice at angiogenic islets stage. It was found that lactate was up-regulated, while acetate, dimethylamine, taurine, glucose and myo-inositol were decreased in the serum samples at 8 weeks (Fig. 5B1). The same OPLS-DA strategy was also used to the metabonome between 3 weeks and 10 weeks, which showed the clear differentiation between them. In addition to the metabolites changes mentioned above, a series of new metabolites with notable changes appeared at the early stage of tumor, including alanine, methionine, citrate and choline. The variation trend of metabolites contributing to the favorable classification between 3 weeks and 14 weeks (Fig. 5B3) were generally in consistent with the changes at 10 weeks, except the changes of glutamate and glycine at 14 weeks. It was noted from the scores plots that the differentiation from the samples at 14 weeks became greater with the aggravation of pathological progression, which indicated that more important metabolites variations appeared with tumor development. The statistical analysis results were showed in table 2. It was observed that changes in lactate, dimethylamine and myo-inositol started to change with statistical significance at 8 weeks, when angiogenic islets emerged. The significantly increased methionine, citrate, choline, and the decreased acetate, taurine and glucose firstly appeared at the early stage of pancreatic islet $\beta$ cell tumor. Therefore, these metabolites could be regarded as potential biomarkers of tumorigenesis. Moreover, the emerging of statistically elevated alanine and glutamate, as well as the decreased glycine were metabolic characteristics for invasive carcinoma in Rip1-Tag2 mice.

Table 2. The potential biochemical markers were statistically analyzed at different stages of Ripl-Tag2 mice.

\begin{tabular}{lllll}
\hline Metabolites & $\delta 1 \mathrm{H}(\mathrm{ppm})$ & 8 week & 10 week & 14 week \\
\hline Lac & $1.33,4.11$ & $\uparrow^{*}$ & $\uparrow^{* *}$ & $\uparrow^{* * *}$ \\
Ala & 1.48 & - & - & $\uparrow^{* *}$ \\
Ace & 1.92 & - & $\downarrow^{*}$ & $\downarrow^{* *}$ \\
Met & 2.13 & - & $\uparrow^{*}$ & $\uparrow^{*}$ \\
Glu & 2.14 & - & - & $\uparrow^{* *}$ \\
Cit & $2.52,2.72$ & - & $\uparrow^{*}$ & $\uparrow^{* *}$ \\
DMA & 2.71 & $\downarrow^{*}$ & $\downarrow^{*}$ & $\downarrow^{* * *}$ \\
Cho & 3.20 & - & $\uparrow^{*}$ & $\uparrow^{* *}$ \\
Tau & $3.26,3,43$ & - & $\downarrow^{* *}$ & $\downarrow^{* * *}$ \\
Glc & $3.40-3.90$ & - & $\downarrow^{*}$ & $\downarrow^{* *}$ \\
Gly & 3.57 & - & - & $\downarrow^{* *}$ \\
MI & $3.53,3.63$ & $\downarrow^{*}$ & $\downarrow^{* *}$ & $\downarrow^{* * *}$ \\
\hline
\end{tabular}

$\uparrow$, denotes increased changes; $\downarrow$, denotes decreased changes; -, no statistical change; ${ }^{*}$ in comparison with Rip1-Tag2 mice at 3 weeks. ${ }^{*} p<0.05 ;{ }^{* * *} p<0.01$; ${ }^{* * * *} p<0.001$. 

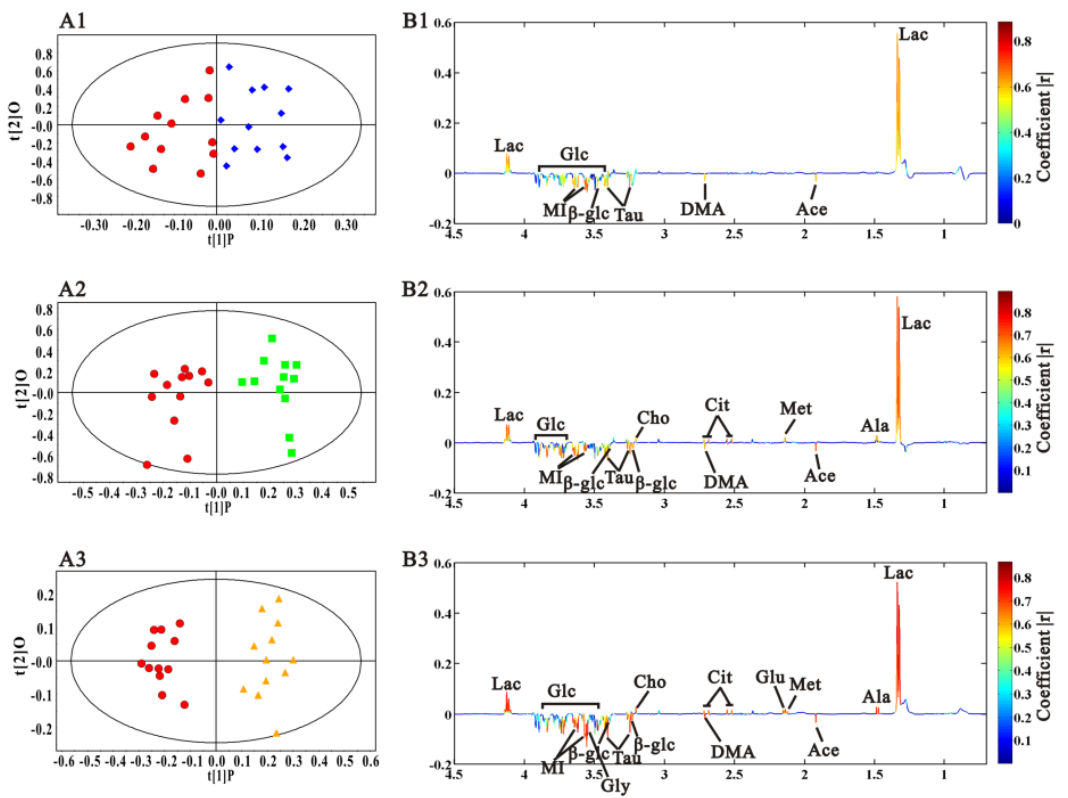

Fig. 5 OPLS-DA analysis results of sera 'H CPMG NMR data in Ripl-Tag2 mice at 3 weeks $(\bullet), 8$ weeks $(\bullet), 10$ weeks $(\boldsymbol{\bullet})$ and 14 weeks $(\boldsymbol{\Delta})$. A, scores plots; $B$, coefficient-coded loadings plots. A1, B1: 8 weeks vs 3 weeks, $R^{2} X=68.5 \%, Q^{2} Y=57.3 \% ; A 2, B 2: 10$ weeks vs 3 weeks, $R^{2} X=76.1 \%, Q^{2} Y=69.3 \% ; A 3$, B3: 14 weeks vs 3 weeks, $R^{2} X=89.5 \%, Q^{2} Y=80.7 \%$.

\section{Discussion}

In this study, metabonomics is first used to explore the association between the development of pancreatic islet $\beta$ cell carcinoma and metabonomic disorders, and discover the metabolic phenotype associated with SV 40 gene transplantation in Rip1-Tag2 mice. Therefore, serum metabonomics was performed to screen biochemical markers for the development of pancreatic islet $\beta$ cell tumor, which will be valuable for the early diagnosis of insulinomas.

From the histopathological characteristics shown in Fig. 1, we could find the noticeable progression of tumor development, and the histopathological changes in every stage showed great reproducibility and were consistent with the previous results $[22,24$, 25]. Fig. 4 demonstrated the metabolic progression trajectory from 3 weeks to 14 weeks, which revealed the serum metabolic changes with the tumor development. However, the samples at 3 weeks and 5 weeks were inseparable due to few metabolic differences between normal and proliferation stages, which may be a result of slight proliferation of pancreatic islet $\beta$ cells at 5 weeks. Multivariate data analyses were performed to identify the metabonomic biomarkers for characterizing the different stages of tumor development. By comparing with sera metabonomic profiles in different types of tumor [28-33], it was found that the increased methionine, and decreased DMA, taurine, glycine and MI were unique characteristics of the serum metabolic profiles in Rip1-Tag2 mice. A general metabolic pathway was drawn according to the changed metabolites (Fig. 6), in which mechanism of glucose, lipid, choline and amino acid were obviously displayed and the mutual transformations between each other were shown in general.

Glucose metabolism is important in the energy supply of whole body. The reduced level of glucose and the elevated lactate were observed in Rip1-Tag2 mice compared to C57 mice, from which we speculated that transplantation of SV 40 gene induced the glucose metabolism changes. Meanwhile, we also found that the level of lactate increased throughout the whole stages of pathological progress in Rip1-Tag2 mice with an accentuated trend (shown in Fig. 5). The metabolic change of glucose and lactate may indicate the time-dependent activation of anaerobic glycolysis, which provided the most energy to meet the energy requirement of the whole body. Furthermore, the increased citrate, one of the most important intermediates in TCA cycle, was also observed during the tumor progression from 10 week to 14 weeks. The accumulated citrate may suggest the disturbance in energy metabolism and the mitochondrial dysfunction as the progression advanced. Insulin, serving as a messenger, can promote muscle and adipose tissue to uptake glucose and provide convenience for anaerobic and aerobic glycolysis [18]. As the increased secretion of insulin in Rip1-Tag2 mice along with the progression advanced, it was found that both the anaerobic glycolysis and aerobic oxidation were enhanced, so the reduced glucose and accumulated of lactate and citrate were observed sub- 
sequently. Moreover, the emerging of increased lactate firstly appeared at angiogenic islets stage, which possibly suggested that excessive lactate from glycolysis as a result of increased energy demand at angiogenic islets stage. Hypoglycemia is usually common in insulinoma, and the reason accounting for this phenomenon is probably hypersecretion of insulin [34] and the activated glucose metabolism from great energy requirement. The increased lactate was also found in esophageal cancer, which demonstrated the activated glucose metabolism by way of glycolysis from aggressive proliferation of tumor cells [31]. However, it was found that the down-regulated TCA metabolism existed in colorectal cancer [33], a possible result of different type of tumor.

Choline, involved in the phospholipid metabolism, has ever been identified as a common metabolic change in tumorigenic development [35, 36]. In this study, the up-regulated of choline was one of the serum metabolic characteristics in Rip1-Tag2 mice associated with SV 40 gene. The significant increase of choline was also found at the tumor formation and invasion metastasis stage, which revealed the metabolic change of choline is an early biomarker for tumor formation and development for pancreatic islet $\beta$ cell carcinoma. The possible molecular mechanisms for choline metabolism may include up-regulated choline kinase [37], accelerated choline transportation [38] and elevated PLD [39] and PLA2 activity [40]. Meanwhile, coupling with the involvement of the tumor growth factors, oncogenes and chemical carcinogens, the activity of choline kinase was enhanced throughout the tumor progression [41]. The role of choline kinase has been demonstrated by many studies, one of which had reported that the choline kinase was over expressed in breast tumor in contrast to normal breast and it had been evidenced to have great association with breast tumor stage [42]. However, there is a decreased level in choline in ovarian cancer, which revealed the disturbance of choline metabolism [28]. Microbial flora was possibly involved in the choline metabolism from the decreased dimethylamine, as a choline metabolite mediated by microbiota. Dimethylamine started to reduce at the angiogenic islets stage, and continuously decreased at tumor formation and invasive carcinoma stage of Rip1-Tag2 mice. This result revealed the increased choline level as a result of the reduced metabolism and activated synthesis of choline. Moreover, the change of choline metabolism at angiogenic islets stage is prior to the change of synthesis pathway. Additionally, the decrease of dimethylamine possibly suggested that gut microbiota disorders was involved in the progression of pancreatic islet $\beta$ cell tumor. However, this needs to be testified by further research.

Insulin is an essential substance to supply amino acids for gluconeogenesis. Study had reported that impaired insulin secretion could directly promote the gluconeogenesis and induce the up-regulation of glucose [43]. The amino acids of methionine, glutamate, and alanine were markedly increased at tumor stage in Rip1-Tag2 mice. The excessive secretion of insulin probably restrained the gluconeogenesis and promoted the transformation from glucose to amino acids. In addition, amino acid transporters were reported to be up-regulated in cancer cells, which could contribute to the accumulation of amino acids [44]. The increased amino acids also reflected the fact that the structural proteins were highly demanded during tumor cells proliferation and apotosis.

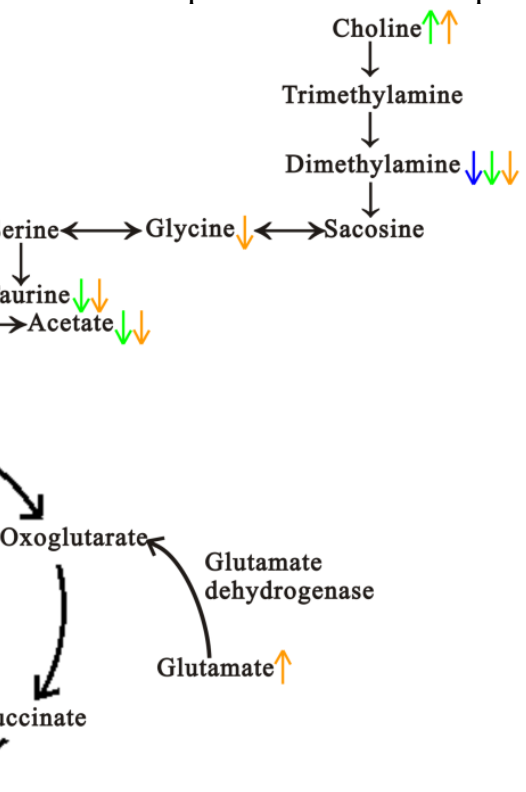

Fig. 6 A summary of the biochemical pathway changes in the pathological progression of Rip1-Tag2 transgenic mice. Blue represents the changes at 8 weeks, green represents the changes at 10 weeks and orange represents the changes at 14 weeks. 
Taurine, a sulfur-containing amino acid, also showed significant decrease in the process of tumor growth. In previous studies, taurine has been regarded as biomarker for bladder carcinoma [45] and endometrial cancer [46], as well as a stable marker for tumor cell apoptosis in astrocytomas of human [47]. In our present study, we found the continuously decreased taurine in sera samples of Rip1-Tag2 mice, which was in consistent with the result in patients of breast cancer [48]. Diverse function of taurine in the body had been explored, which included intracellular osmoregulation [49], antioxidant activity [50] and bile acid formation [51]. The reduced taurine possibly indicated the disorder in one or all of the mentioned functions.

Myo-inositol, as a source of second messengers, is a precursor in the phosphatidylinositol metabolism. In this study, the level of myo-inositol was found to decline constantly in the progression of the pancreatic islet $\beta$ cell carcinoma. This result implied myo-inositol might be a biomarker in the hemorrhagic pancreas period, and it might correlate with the malignant degree of the pancreatic islet $\beta$ cell carcinoma.

\section{Conclusion}

In summary, our findings presented that NMR-based metabonomics has the power to identify biomarkers that correlated with the tumor progression in Rip1-Tag2 mice. There are early decreases in myo-inositol and dimethylamine, and increase in lactate concentrations that occur at the angiogenic islets stage. The activated energy and amino acid metabolism were found at the early stage of tumor formation. The notable changes in alanine, glutamate and glycine were characteristics in metabolic profiles at invasive carcinoma stage. Additionally, this approach has been extended to determine the metabolic phenotype associated with gene alterations in Rip1-Tag2 mice, which potentially regarded as surrogates for the genetic event.

\section{Supplementary Material}

Fig. S1 - S2. http://www.ijbs.com/v11p0595s1.pdf

\section{Acknowledgements}

The study was supported by the National Natural Science Foundation of China $(31271455,31471290$, 21005022, 31200861, 31200896, 31100852).

\section{Abbreviations}

EUS, endoscopic ultrasonography; MRI, magnetic resonance imaging; $\mathrm{CT}$, computed tomography; CPMG, Carr-Purcell-Meiboom-Gill; COSY, correlation spectroscopy; TOCSY, total correlation spectroscopy; HSQC, heteronuclear single quantum coherence spectroscopy; PCA, principal component analysis; OPLS-DA, orthogonal partial least-squaresdiscriminant analysis.

\section{Competing Interests}

The authors have declared that no competing interest exists.

\section{References}

1. Abood GJ, Go A, Malhotra D, et al. The surgical and systemic management of neuroendocrine tumors of the pancreas. Surg Clin North Am. 2009; 89: 249-66.

2. Fendrich V, Waldmann J, Bartsch DK, et al. Surgical management of pancreatic endocrine tumors. Nat Rev Clin Oncol. 2009; 6: 419-28.

3. Service FJ, McMahon MM, O'Brien PC, et al. Functioning insulinoma--incidence, recurrence, and long-term survival of patients: a 60-year study. Mayo Clin Proc. 1991; 66: 711-9.

4. Tucker ON, Crotty PL, Conlon KC. The management of insulinoma. Br J Surg. 2006; 93: 264-75.

5. Doppman JL, Chang R, Fraker DL, et al. Localization of insulinomas to regions of the pancreas by intra-arterial stimulation with calcium. Ann Intern Med. 1995; 123: 269-73.

6. Günther RW, Klose KJ, Rückert $\mathrm{K}$, et al. Localization of small islet-cell tumors.Preoperative and intraoperative ultrasound, computed tomography, arteriography, digital subtraction angiography, and pancreatic venous sampling. Gastrointest Radiol. 1985; 10: 145-52.

7. McLean AM, Fairclough PD. Endoscopic ultrasound in the localisation of pancreatic islet cell tumours. Best Pract Res Clin Endocrinol Metab. 2005; 19: 177-93.

8. Gouya H, Vignaux O, Augui J, et al. CT, endoscopic sonography, and a combined protocol for preoperative evaluation of pancreatic insulinomas. AJR Am J Roentgenol. 2003; 181: 987-92.

9. Richards ML, Gauger PG, Thompson NW, et al. Pitfalls in the surgical treatment of insulinoma. Surgery. 2002; 132: 1040-9.

10. Holmes E, Bonner FW, Sweatman BC, et al. Nuclear magnetic resonance spectroscopy and pattern recognition analysis of the biochemical processes associated with the progression of and recovery from nephrotoxic lesions in the rat induced by mercury(II) chloride and 2-bromoethanamine. Mol Pharmacol. 1992; 42: 922-30.

11. Nicholson JK, Lindon JC, Holmes E. 'Metabonomics': understanding the metabolic responses of living systems to pathophysiological stimuli via multivariate statistical analysis of biological NMR spectroscopic data. Xenobiotica. 1992; 29: 1181-9.

12. Gao HC, Lu Q, Liu X, et al. Application of $1 \mathrm{H}$ NMR-based metabonomics in the study of metabolic profiling of human hepatocellular carcinoma and liver cirrhosis. Cancer Sci. 2009; 100: 782-5.

13. Asiago VM, Alvarado LZ, Shanaiah N, et al. Early detection of recurrent breast cancer using metabolite profiling. Cancer Res. 2010; 70: 8309-18.

14. Sreekumar A, Poisson LM, Rajendiran TM, et al. Metabolomic profiles delineate potential role for sarcosine in prostate cancer progression. Nature. 2009; 457: 910-4

15. Bathe OF, Shaykhutdinov R, Kopciuk K, et al. Feasibility of identifying pancreatic cancer based on serum metabolomics. Cancer Epidemiol Biomarkers Prev. 2011; 20: 140-7.

16. Zira AN, Theocharis SE, Mitropoulos D, et al. $1 \mathrm{H}$ NMR metabonomic analysis in renal cell carcinoma: a possible diagnostic tool. J Proteome Res. 2010; 9: 4038-44.

17. Ludwig C, Ward DG, Martin A, et al. Fast targeted multidimensional NMR metabolomics of colorectal cancer. Magn Reson Chem. 2009; 47: S68-73.

18. Zhang $\mathrm{XY}$, Wang $\mathrm{YL}, \mathrm{Hao} \mathrm{FH}$, et al. Human serum metabonomic analysis reveals progression axes for glucose intolerance and insulin resistance statuses. J Proteome Res. 2009; 8: 5188-95.

19. Chun MG, Mao JH, Chiu CW, et al. Polymorphic genetic control of tumor invasion in a mouse model of pancreatic neuroendocrine carcinogenesis. Proc Natl Acad Sci USA. 2010; 107: 17268-73.

20. Nozawa H, Chiu C, Hanahan D. Infiltrating neutrophils mediate the initial angiogenic switch in a mouse model of multistage carcinogenesis. Proc Natl Acad Sci USA. 2006; 103: 12493-8.

21. Müller-Hermelink N, Braumüller H, Pichler B, et al. TNFR1 signaling and IFN-gamma signaling determine whether $\mathrm{T}$ cells induce tumor dormancy or promote multistage carcinogenesis. Cancer Cell. 2008; 13: 507-18.

22. Bergers G, Javaherian K, Lo KM, et al. Effects of angiogenesis inhibitors on multistage carcinogenesis in mice. Science. 1999; 284: 808- 12.

23. Tuveson D, Hanahan D. Translational medicine: Cancer lessons from mice to humans. Nature. 2011; 471: 316-7.

24. Hanahan D. Heritable formation of pancreatic beta-cell tumors in transgenic mice expressing recombinant insulin/simian virus 40 oncogenes. Nature. 1985; 315: 115-22.

25. Li JC, Yang YX, Zhou XM, et al. Micrometastasis expressing insulin arise in lung and spleen at advanced stage of Rip1-Tag2 transgenic mice. Int J Biol Sci. 2014; 10: 136-41. 
26. Tang HR, Wang YL, Nicholson JK, et al. Use of relaxation-edited one-dimensional and two dimensional nuclear magnetic resonance spectroscopy to improve detection of small metabolites in blood plasma. Anal Biochem. 2004; 325: 260-72.

27. Nicholson JK, Foxall PJ, Spraul M, et al. $750 \mathrm{MHz} 1 \mathrm{H}$ and $1 \mathrm{H}-13 \mathrm{C}$ NMR spectroscopy of human blood plasma. Anal Chem. 1995; 67: 793-811.

28. Garcia E, Andrews C, Hua J, et al. Diagnosis of early stage ovarian cancer by $1 \mathrm{H}$ NMR metabonomics of serum explored by use of a microflow NMR probe. J Proteome Res. 2011; 10: 1765-71.

29. Yonezawa K, Nishiumi S, Kitamoto-Matsuda J, et al. Serum and tissue metabolomics of head and neck cancer. Cancer Genomics Proteomics. 2013; 10: 233-8.

30. Tiziani S, Lopes V, Günther UL. Early stage diagnosis of oral cancer using $1 \mathrm{H}$ NMR-based metabolomics. Neoplasia. 2009; 11: 269-76.

31. Zhang $\mathrm{X}, \mathrm{Xu} \mathrm{L}$, Shen J, et al. Metabolic signatures of esophageal cancer: NMR-based metabolomics and UHPLC-based focused metabolomics of blood serum. Biochim Biophys Acta. 2013; 1832: 1207-16.

32. Li $Y$, Song $X$, Zhao $X$, et al. Serum metabolic profiling study of lung cancer using ultra highperformance liquid chromatography/quadrupole time-of-flight massspectrometry. J Chromatogr B Analyt Technol Biomed Life Sci. 2014; 966: 147-53.

33. Bertini I, Cacciatore S, Jensen BV, et al. Metabolomic NMR fingerprinting to identify and predict survival of patients with metastatic colorectal cancer. Cancer Res. 2012; 72: 356-64.

34. Iglesias P, Díez JJ. Management of endocrine disease: a clinical update on tumor-induced hypoglycemia. Eur J Endocrinol. 2014; 170: R147-57.

35. Herminghaus S, Pilatus U, Möller-Hartmann W, et al. Increased choline levels coincide with enhanced proliferative activity of human neuroepithelial brain tumors. NMR Biomed. 2002; 15: 385-92.

36. Stanwell P, Gluch L, Clark D, et al. Specificity of choline metabolites for in vivo diagnosis of breast cancer using $1 \mathrm{H}$ MRS at 1.5 T. Eur Radiol. 2005; 15: 1037-43.

37. Ramírez de Molina A, Rodríguez-González A, Gutiérrez R, et al. Overexpression of choline kinase is a frequent feature in human tumor-derived cell lines and in lung, prostate, and colorectal human cancers. Biochem Biophys Res Commun. 2002; 296: 580-3.

38. Katz-Brull R, Degani H. Kinetics of choline transport and phosphorylation in human breast cancer cells; NMR application of the zero trans method. Anticancer Res. 1996; 16:1375-80.

39. Noh DY, Ahn SJ, Lee RA, et al. Overexpression of phospholipase D1 in human breast cancer tissues. Cancer Lett. 2000; 161:207-14.

40. Guthridge CJ, Stampfer MR, Clark MA, et al. Phospholipases A2 in ras-transformed and immortalized human mammary epithelial cells. Cancer Lett. 1994; 86:11-21.

41. Ackerstaff E, Glunde K, Bhujwalla ZM. Choline phospholipid metabolism: a target in cancer cells? J Cell Biochem. 2003; 90: 525-33.

42. Ramírez de Molina A, Gutiérrez R, Ramos MA, et al. Increased choline kinase activity in human breast carcinomas: clinical evidence for a potential novel antitumor strategy. Oncogene. 2002; 21: 4317-22.

43. Krebs M, Brehm A, Krssak M, et al. Direct and indirect effects of amino acids on hepatic glucose metabolism in humans. Diabetologia. 2003; 46: 917-25.

44. Hirayama A, Kami K, Sugimoto M, et al. Quantitative metabolome profiling of colon and stomach cancer microenvironment by capillary electrophoresis time-of-flight mass spectrometry. Cancer Res. 2009; 69: 4918-25.

45. Srivastava S, Roy R, Singh S, et al. Taurine-a possible fingerprint biomarker in non-muscle invasive bladder cancer: A pilot study by $1 \mathrm{H}$ NMR spectroscopy. Cancer Biomark. 2010; 6: 11-20.

46. Agouza IM, Nashar DE. Serum taurine as a marker of endometrial cancer. Open Women's Health J. 2011; 5:1-6.

47. Opstad KS, Bell BA, Griffiths JR, et al. Taurine: a potential marker of apoptosis in gliomas. Br J Cancer. 2009; 100: 789-94.

48. EI Agouza IM, Eissa SS, El Houseini MM, et al. Taurine: a novel tumor marker for enhanced detection of breast cancer among female patients. Angiogenesis. 2011; 14: 321-30.

49. Semenova NA, Dydykina IY, Dederer LY, et al. The use of $1 \mathrm{H}-\mathrm{NMR}$ spectroscopy for predicting the efficiency of neoadjuvant chemotherapy of breast cancer. Bull Exp Biol Med. 2000; 130: 701-4.

50. Schaffer SW, Azuma J, Mozaffari M. Role of antioxidant activity of taurine in diabetes. Can J Physiol Pharmacol. 2009; 87: 91-9.

51. Wesseling S, Koeners MP, Joles JA. Taurine: red bull or red herring? Hypertension. 2009; 53: 909-11. 